Gennady Zaikov and Marina Artsis

\title{
DEGRADATION OF POLYMERS IN AGgRESSIVE MEDIA. KINETIC APPROACH
}

\author{
N.M. Emanuel Institute of Biochemical Physics, Russian Academy of Sciences \\ 4 Kosygin str., 119334 M oscow, Russia \\ chembio@sky.chph.ras.ru
}

Received: September 01, 2008

(C) Zaikov G., Artsis M., 2009

\begin{abstract}
The degradation of polymers in aggressive media is a complex physico-chemical process including adsorption, diffusion and the dissociation of chemically unstable bonds. The course of degradation has a number of special features, which are linked both with the specific structure of polymeric materials and with specific kinetics of reactions in solids.
\end{abstract}

Key words: degradation, stabilization, aggressive media, carbochain and heterochain polymers, chemical kinetics.

\section{Fundamental Kinetic Equations}

In the course of degradation of polymers in aggressive media there are the following fundamental processes:

1) adsorption of the aggressive medium on the surface of the polymer article;

2) diffusion of the aggressive medium within the polymer article;

3 ) chemical reaction of the aggressive medium with chemically unstable groups of the polymer;

4) diffusion of degradation products to the surface of the polymer article;

5) desorption of degradation products from the surface of the polymer article [1-10].

A mathematical consideration of these processes is very difficult, and, accordingly, it is usually assumed that one or at most two stages are slow in comparison with the others, and consequently limit the course of the whole degradation process. The first and the fifth stages usually take place more rapidly than the second, the third and the fourth ones.

The diffusion of degradation products to the surface of the polymer article usually plays insignificant role in the assessment of the change in the service properties of polymer articles in the course of degradation, but it may restrict, for instance, the process of biodegradation of a polymer within a living organism. In the course of chemical degradation the polymer and the aggressive medium (liquid or gas) are in separate phases. The chemical reaction between the aggressive medium and chemically unstable groups in the polymer may take place either at the interface phase or within the polymer phase (components of the aggressive medium which are dissolved in the polymer take part in the reaction).

Regarding these two phases as a single closed system, the rate of the chemical reaction $w$ may be expressed as $\frac{d n}{V d t}$, where $n$ is the number of groups which have decomposed at time $t$, and $V$ is the volume of the polymer.

In the first case $w \approx \frac{S}{V}$, where $S$ is the surface area of the polymer article and, in the second case, this relationship is more complex. Let us consider it in details. Assuming that the law of the mass action is observed, the volume of the polymer remains practically unchanged during the course of degradation and the polymer is isotropic in properties; then we can write the equation for the rate of decomposition of the chemically unstable groups as follows:

$$
w=\frac{d c_{n}}{d t}=k\left(c_{n}^{0}-c_{n}\right) c_{\text {cat }} c_{\text {solv }}
$$

where $c_{n}^{0}$ is the initial concentration of chemically unstable groups in the polymer; $c$ is the concentration of decomposed groups; $c_{\text {cat }}$ is the concentration of the catalyst in the polymer; $c_{\text {solv }}$ is the concentration of the solvent in the polymer; $k$ is the rate constant of decomposition of the unstable groups.

The concentration of the catalyst in the polymer, e.g. acid or base, can be found from the equation (2).

$$
\frac{d c_{c a t}}{\partial t}=D_{c a t} \nabla^{2} c_{c a t}-\sum_{i} c_{c a t} c_{i} k_{i}
$$

where $\nabla$ is the Laplace operator; $c_{i}$ is the concentration of functional groups in the polymer which are capable of taking part in a complex formation or substitution reaction; 
$k_{i}$ is the rate constant of the complex formation or substitution reaction of the catalyst with functional groups of the polymer.

The second term on the right-hand side of Eq. (2) takes into account the possibility of such reactions as protonation, interaction with a hydroxide ion, and so forth.

If the solvent is involved in decomposition of chemically unstable groups in the polymer, for instance the removal of water in hydrolysis reactions, then the solvent concentration may be found from the equation (3).

$$
\frac{d c_{\text {solv }}}{\partial t}=D_{\text {solv }} \nabla^{2} c_{\text {solv }}-k\left(c_{n}^{0}-c_{n}\right) c_{\text {cat }} c_{\text {solv }}
$$

While writing this equation it is necessary to justify a number of assumptions.

First, the polymer-aggressive medium system is, as a rule, very much diluted in relation to the aggressive medium, i.e. it may be assumed that $D_{\text {cat }}$ and $D_{\text {solv }}$ do not depend on the concentration of the corresponding components in the polymer. If the aggressive medium is soluble in the polymer to any considerable extent, it becomes necessary to use an equation which takes into account the relationship between $D$ and the diffusant concentration.

Second, the value of $k$ is constant. This is correct when the aggressive medium has a low solubility in the polymer. In general, the influence of the aggressive medium on $k$ can be reduced, as a first approximation, to the influence of the solvent on the rate of the chemical reaction, which can be predicted on the basis of existing theories [11].

Third, the decomposition reaction of chemically unstable groups is practically irreversible. This condition is realized when degradation takes place under some particular reaction conditions to quite low degrees of dissociation $(<0.05)$. In this case the concentration of chemically unstable groups changes only slightly and $c_{n}^{0}-c_{n} \approx c_{n}^{0}$.

Thus, determining the rate of decomposition of chemically unstable groups in a polymer under the action of aggressive media means the joint solution of Eqs. (1)-(3).

Usually the following boundary conditions are assumed:

- the concentration of substances diffusing to the surface of a polymer article is a constant value (in practice this condition is fulfilled with a rapid flow over the article of a stream of solution with the constant concentration of the aggressive medium);

- the concentration of substances diffusing to the surface of the polymer article is a function of time, and the dependence of the concentration on time is expressed by one of the adsorption equations;

- the concentration of substances diffusing to the surface of the polymer article is determined by some particular law of mass transfer, the simplest being $j=\beta\left(c_{\text {surf }}-c_{p}\right)$, where $j$ is the flow of substance diffusing; $\beta$ is the mass transfer coefficient; $c_{\text {sur }}$ and $c_{p}$ are the concentration of the substance on the surface and within the volume of the solution of the aggressive medium, respectively.

The majority of polymer articles may, to a first approximation, be regarded as simple geometrical bodies.

We can take a parallelepiped as a model of polymer films and coatings and a cylinder as a model of a filament and so forth.

We shall now consider the solution of Eqs. (1)-(3) for a parallelepiped and a cylinder.

\section{Macrokinetics of Chemical Degradation}

The process of degradation may take place in various regions, depending on the ratio of rates of the diffusion process and of the chemical reaction.

The rate of diffusion of the aggressive medium is commensurate with the rate of the chemical reaction, and degradation takes place in a particular reaction zone which size increases with time and ultimately reaches the limits of the dimensions of the polymer article, i.e. the reaction takes place under internal diffusion-kinetic conditions.

The rate of diffusion of the aggressive medium considerably exceeds that of the chemical reaction. After dissolution of the aggressive medium in the polymer is finished, the degradation takes place over the total volume of the polymer, i.e. under internal kinetic conditions.

The rate of diffusion of the aggressive medium is considerably lower than that of the chemical reaction. In this case degradation takes place in a particular thin reaction surface layer or, as it is usually put, from the surface of the polymer article, i.e. under external diffusion-kinetic conditions.

\subsection{Internal Diffusion-Kinetic Conditions}

The joint solution of Eqs. (1) - (3), even with the above assumptions, is a difficult mathematical problem and is possible only by using a computer. While considering the diffusion of the aggressive media in polymers, it has been established that the diffusion of the catalyst (acid or base) and of the solvent takes place at an identical rate on a single front and that the diffusion of the solvent is at a considerably higher rate than that of the catalyst.

The first variant occurs, as a rule, in the diffusion of the aggressive media in hydrophilic polymers and in the diffusion of acids and bases with a high vapor pressure in hydrophobic polymers.

The second variant occurs in the diffusion of acids and bases with a low vapor pressure in hydrophobic polymers.

These patterns of behavior simplify the solution of the problem, since, in the first case, the diffusion of the catalyst and solvent can be characterized by a single diffusion coefficient $D_{\text {cat }}=D_{\text {solv }}$. In the second case, $D_{\text {soly }}$ $>D_{c a t}$, and it can be reckoned that the concentration of 
the solvent in the reaction zone of the polymer article becomes constant after a certain time and equals to its solubility $c_{\text {solv }}^{0}$, i.e.

$$
\frac{\partial c_{\text {solv }}}{\partial t}=0 \text { when } c_{\text {solv }}=c_{\text {solv }}^{0} .
$$

Thus, the problem is simplified and is reduced to the solving of Eqs. (1) and (2), of which, taking into account the relationship $c_{n}^{0}-c_{n} \approx c_{n}^{0}$, the second takes the form

$$
\frac{d c_{n}}{d t}=k_{e f f} c_{c a t}
$$

where

$$
k_{e f f}=k c_{n}^{0} c_{\text {solv }}^{0} .
$$

In Eq. (2), for simplicity, we restrict ourselves to one term and make $c=c_{i}$.

Depending on whether or not the combination of the catalyst with functional groups of the polymer takes place, we may obtain two equations for the change in the number of macromolecular scissions in the course of degradation.

$\underline{K}_{p} \rightarrow \infty$, i.e. the functional groups present in the polymer formed in the course of degradation react with the catalyst practically irreversibly. Bearing in mind that these reactions (e.g., protonation or complex formation) proceed far more rapidly than degradation reactions, Eq. (2) takes the form

$$
\frac{\partial c_{c a t}}{\partial t}=D_{c a t} \nabla^{2} c_{c a t}-k_{e f f} c_{c a t}
$$

The solution of this equation was first achieved by Danckwerts [12], on the basis of the solution of the analogous problem of thermal conductivity considered by Carslaw and Jaeger [13]. The solution of Eq. (5) for a parallelepiped one of which dimensions $(l)$ is much less than the other two with the boundary condition $c_{c a t}=c_{c a t}^{0}$ with $x=0$ and $l$ with $t \rightarrow 0$ and the initial condition $c_{\text {cat }}=0$ with $t=0$ and $0 \leq x \leq l$ takes the form

$$
\begin{gathered}
c_{c a t}(x, t)=c_{\text {cat }}^{0} \times \\
\left\{1-\frac{4}{\pi} \sum_{m=0}^{\infty} \frac{\sin b_{m} x}{\left.(2 m+1)\left(b_{m}^{2} D_{c a t}+1\right)^{\left[k_{e f f}\right.}+b_{m}^{2} D_{c a t} \exp -\left(b_{m}^{2} D_{c a t}+k_{e f f}\right) t\right]}\right\}
\end{gathered}
$$

where $b_{m}=\frac{\pi(2 m+1)}{l} ; c_{c a t}^{0}$ is the solubility of the catalyst in the polymer.

Substituting Eq. (6) into (4), and carrying out double integration from 0 to $t$ and from 0 to $l / 2$, we get

$$
\begin{gathered}
c_{n}=k_{e f f} c_{c a t}^{0} t \times \\
\times\left\{1-\frac{8}{\pi^{2}} \sum_{m=0}^{\infty} \frac{k_{e f f}\left(b_{m}^{2} D_{c a t}+k_{e f f}\right)+b_{m}^{2} D_{c a t}\left[1-\exp -\left(b_{m}^{2} D_{c a t}+k_{e f f}\right) t\right]}{(2 m+1) b_{m}\left(b_{m}^{2} D_{c a t}+k_{e f f}\right)^{2} l t}\right\}
\end{gathered}
$$

Examination of Eq. (7) shows that if $b_{m}^{2} D_{\text {cat }}>k_{\text {eff }}$, it is sufficient to limit oneself to the first term of the series. A detailed consideration of this case will be given below.

Solution of Eq. (5) for a cylinder of radius $r$ much less than the length $l$ with the boundary conditions $c_{c a t}=c_{c a t}^{0}$ with $x=r$ with $t>0$ and the initial condition $c_{\text {cat }}=0$ with $t=0$ and $x \leq r$, takes the form

$$
\begin{gathered}
c_{c a t}(x, t)=c_{\text {cat }}^{0} \times \\
\left\{1-\frac{2}{r} \sum_{n=1}^{\infty} \frac{\left[k_{\text {eff }}+D_{\text {cat }} \frac{\mu_{n}^{2}}{r^{2}} \exp -\left(D_{\text {cat }} \frac{\mu_{n}^{2}}{r^{2}}+k_{\text {eff }}\right)\right] J_{0}\left(\frac{x}{r} \mu_{n}\right)}{\left(D_{c a t} \frac{\mu_{n}^{2}}{r^{2}}+k_{\text {eff }}\right) \frac{\mu_{n}}{r} J_{1}\left(\mu_{n}\right)}\right\}
\end{gathered}
$$

where $J_{0}$ and $J_{1}$ are Bessel functions of the first kind of zero and first order, respectively; and $\mu_{n}$ are the roots of the Bessel function.

An expression for $c_{n}$ is obtained from Eqs. (4) and (8) after integration from 0 to $t$ and from 0 to $r$ :

$$
c_{n}=k_{\text {eff }} c_{c a t}^{0} \sum_{n=1}^{\infty} \frac{D_{c a t}}{r^{2} z}\left[t-\frac{1-\exp (-z t)}{z}\right]
$$

where $z=\frac{D_{c a t}}{r^{2}}+k_{\text {eff }}$.

For the initial time of degradation, if $\exp (-z t)>0.9$, we can use the approximated equation (10) for calculations:

$$
c_{n} \approx k_{\text {eff }} c_{c a t}^{0}\left[\frac{8}{3 \pi^{1 / 2}} \cdot \frac{D_{c a t}^{1 / 2}}{r} t^{3 / 2}-\frac{D_{c a t} t^{2}}{2 r^{2}}-\frac{2}{15 \pi^{1 / 2}} \cdot \frac{D_{c a t}^{1 / 2}}{r}\left(\frac{D_{c a t}}{r^{2}}+k_{\text {eff }}\right) f^{5 / 2}\right]
$$

If $\exp (-z t)<0.1$, then Eq. (9) takes the form

$$
c_{n} \approx k_{e f f} c_{c a t}^{0} \sum_{n=1}^{\infty} \frac{D_{c a t}}{z r^{2}}\left(t-\frac{1}{z}\right)
$$

If $\frac{D_{c a t} \mu_{n}^{2}}{r^{2}}>k_{e f f}$, then, as in the case considered above, it is sufficient to restrict ourselves to the first term of the series, which simplifies Eqs. (9) and (11).

$K_{p} \rightarrow 0$, i.e., the functional groups in the polymers do not react appreciably with the catalyst. Eq. (2) takes the form of the Pick diffusion equation, the solution of which, for the parallelepiped (film) and cylinder (filament) mentioned above, is discussed in literature.

On substituting the solution into the Fick equation for the parallelepiped in (4) and integrating within the same limits as in Eq. (9), we get 


$$
c_{n}=k_{e f f} c_{c a t}^{0} t\left\{1-\frac{8}{\pi^{2}} \sum_{m=0}^{\infty}\left[1-\exp (2 m+1)^{2} y\right] \frac{1}{(2 m+1)^{4} y}\right\}
$$

where $y=\frac{\pi^{2} D_{c a t} t}{l^{2}}$

With $y<1$, this case is realized in the initial period of degradation for films of any particular thickness

$$
c_{n}=k_{\text {eff }} c_{c a t}^{0} t \frac{8}{\pi^{2}} \varphi(y)
$$

where $\varphi(y)=\sum_{m=0}^{\infty} \frac{\exp -(2 m+1)^{2} y-1+(2 m+1)^{2} y}{(2 m+1)^{4} y}$.

By using a computer there is established a simple relationship $\varphi(y)=0.589 y^{1 / 2}$, i.e.,

$$
c_{n}=\frac{4}{\pi^{1 / 2}} k_{e f f} c_{c a t}^{0} D_{c a t}^{1 / 2} l^{-1} t^{3 / 2}
$$

Eq. (14) is conveniently expressed as follows:

$$
n=\frac{8}{\pi^{1 / 2}} k_{e f f} c_{c a t}^{0} D_{c a t}^{1 / 2} s t^{3 / 2}
$$

Thus, in the initial period of degradation the number of groups which have decomposed depends on the rate constant of the chemical reaction, on the diffusion coefficient, on the solubility of the catalyst, on the surface area of the film and on time.

For a cylinder, after the corresponding operations, we get

$$
c_{n}=k_{e f f} c_{c a t}^{0}\left\{t-\sum_{n=1}^{\infty} \frac{4 r^{2}}{\mu_{n}^{2} D_{c a t}}\left[1-\exp -\left(\frac{D_{c a t}}{r^{2}} \mu_{n}^{2} t\right)\right]\right\}
$$

While solving this equation it is sufficient to restrict oneself to the first term of the series.

For the initial period of degradation with

$$
\begin{gathered}
\left(-\frac{D_{c a t}}{r^{2}} \mu_{1}^{2} t\right)<0.1 \text { it is possible to use the equation } \\
c_{n} \approx k_{\text {eff }} c_{c a t}^{0}\left[\frac{8}{3 \pi^{1 / 2}} \frac{D_{c a t}^{1 / 2}}{r} \mu_{1}^{2} t^{3 / 2}-\frac{D_{c a t} t^{2}}{2 r^{2}}-\frac{2}{15 \pi^{1 / 2}}\left(\frac{D_{c a t}}{r^{2}}\right)^{3 / 2} t^{5 / 2}\right](17) \\
\text { If }\left(-\frac{D_{c a t}}{r^{2}} \mu_{1}^{2} t\right) \leq 0.9 \text {, we get the linear equation } \\
c_{n} \approx k_{\text {eff }} c_{c a t}^{0}\left(t-\frac{4 r^{2}}{\mu_{1}^{2} D_{c a t}}\right)
\end{gathered}
$$

where the intercept on the time axis

$$
t_{0} \approx \frac{4 r^{2}}{\mu_{1}^{4} D_{c a t}}
$$

while the slope is equal to the product $k_{\text {eff }} c_{\text {cat }}^{0}$.

An approximate solution of Eq. (2) for a chemically reversible reaction ( $K_{e q}$ does not approach infinity) is considered in [14].

\subsection{Internal Kinetic Conditions}

The kinetic equation for the parallelepiped may be obtained from Eq. (12) with $\mathbf{y} \gg>1$, and for the

cylinder, from Eq. (16) with $\left(-\frac{D_{c a t}}{r^{2}} \mu_{1}^{2} t\right) \rightarrow 1$.

In both cases,

$$
c_{n}=k_{\text {eff }} c_{\text {cat }}^{0} t
$$

Thus, at fairly low degrees of dissociation the concentration of groups which have decomposed is proportional to the catalyst concentration under saturation conditions.

\subsection{External Diffusion-Kinetic Conditions}

The process of degradation under these conditions takes place in a particular thin reaction surface layer, the dimensions of which usually cannot be determined because of the lack of values for $k_{\text {eff }}$ and $D_{\text {cat }}$. It is usually assumed that this layer is infinitely small and that degradation takes place practically from the surface of the polymer article. In this case

$$
\frac{d n}{d t}=k_{\text {surf }} c_{n(\text { surf })} c_{\text {cat (surf) }} c_{\text {solv(surf })} S
$$

where "surf" indicates that the concentration relates to the polymer-aggressive medium interface.

While solving this equation it is assumed that the polymer is orientated, and that the macromolecules are arranged parallel to the larger part of the interface (e.g., for a filament the curved surface will be the greatest). The concentration of active sites on the surface $c_{n(\text { surf } f}$ for the process in question depends on the type of decomposition of the macromolecules, their packing, the cohesive energy and the solubility of decomposition products (monomers and oligomers) in the surrounding medium.

With this type of degradation within a very short time on the surface of the polymer article a steady-state concentration of active sites $c_{n(\text { surf })}^{0} z^{-1}$ (where $z$ is a constant depending on the factors indicated above) is established. For instance, if decomposition of the 
macromolecules is at a terminal bond, and formed monomers are soluble in the aggressive medium, then $c_{n(\text { surf })} \approx \frac{c_{n(\text { surf })}^{0}}{\bar{P}_{n_{0}}}$ and $k_{\text {surf }}$ is the rate constant of depolymerization. If decomposition of the macromolecules is a random process with subsequent rapid single-act depolymerization, and the degradation products are soluble, then $c_{n(\text { surf })} \approx c_{n(\text { surf })}^{0}$ and $k_{(\text {surf })}$ is the rate constant.

Let us consider how the linear dimensions and mass of polymer articles of different geometrical forms will change with time.

Let us express the degree of dissociation by the number of chemically unstable groups which have decomposed to the maximum possible number of scissions $\left(n_{\infty}\right)$

$$
\alpha=\frac{n}{n_{\infty}}
$$

We can determine the value of $n_{\infty}$ from the formula

$$
n_{\infty}=\frac{m_{0} A}{M}\left[1-\frac{1}{\left(\bar{P}_{n_{0}}-1\right)}\right]
$$

where $M$ is the MM (molecular mass) of the monomer unit.

For quite large $\bar{P}_{n_{0}}$ in formula (23) we can restrict ourselves to the first term. If the degradation products are soluble in the surrounding medium, $\alpha$ may be expressed by the mass of the polymer

$$
\alpha=\left(1-\frac{m}{m_{0}}\right)
$$

In such a case,

$$
\frac{d \alpha}{d t}=\frac{1}{n_{\infty}} \frac{d n}{d t}=-\frac{1}{m_{0}} \frac{d m}{d t}
$$

Substituting (21) into (25), we get

$$
-\frac{d m}{d t}=\frac{M}{A} k_{(\text {surf })} \frac{c_{n(\operatorname{surf})}^{0}}{z} c_{c a t(\operatorname{surf})} c_{\text {solv (surf })} S
$$

For a film of thickness $l$,

$$
m=s l \rho .
$$
follows:

The thickness of the specimen will change as

$$
l=l_{0}-k_{(\text {surf })} \frac{M}{A} c_{n(\text { surf })}^{0} \frac{1}{z \rho} c_{\text {cat (surf })} c_{\text {solv (surf })} S
$$

The time for complete decomposition of the polymer is

$$
\tau=\frac{l_{0} \rho}{k_{(\text {surf })} \frac{M}{A} c_{n(\text { surf })}^{0} \frac{1}{z} c_{\text {cat (surf })} c_{\text {solv (surf })}}
$$

Since the surface area of the polymer article remains practically unchanged right up to high degrees of dissociation, we get from Eq. (28)

$$
m=m_{0}-k_{(\text {surf })} \frac{M}{A} c_{n(\text { surf })}^{0} \frac{1}{z} s c_{\text {cat (surf })} c_{\text {solv (surf })} t
$$

Eqs. (27) and (28) can be expressed by dimensionless parameters

$$
\begin{gathered}
\frac{m}{m_{0}}=1-\frac{t}{\tau} \\
\frac{l}{l_{0}}=1-\frac{t}{\tau}
\end{gathered}
$$

For a filament of radius $r$ we find from Eq. (24)

$$
\alpha=1-\frac{r^{2}}{r_{0}^{2}}
$$

and

$$
\frac{d \alpha}{d t}=\frac{1}{n_{\infty}} \frac{d n}{d t}=-\frac{2 r d r}{r_{0}^{2} d t}
$$

We obtain via corresponding transformations

$$
r=r_{0}-k_{(\text {surf })} \frac{M}{A} c_{n(\text { surf })}^{0} \frac{1}{z \rho} c_{\text {cat (surf })} c_{\text {solv (surf })} t^{t}
$$

From Eq. (34) we determine the change in mass of the polymer with time, using the relationship between the surface area of the side and the volume of the cylinder:

$$
m^{1 / 2}=m_{0}^{1 / 2}-k_{(\text {surf })} \frac{M}{A} c_{n(\text { surf })}^{0} \frac{(\pi l)^{1 / 2}}{(z \rho)^{1 / 2}} c_{\text {cat (surf })} c_{\text {solv (surf })} t^{t}
$$

Using dimensionless parameters, Eqs. (34) and (35) take the following form:

$$
\frac{r}{r_{0}}=1-\frac{t}{\tau}
$$

$$
\frac{m}{m_{0}}=\left(1-\frac{t}{\tau}\right)^{2}
$$


where

$$
\tau=\frac{r_{0} \rho}{k_{(\operatorname{surf})} \frac{M}{A} c_{n(\operatorname{surf})}^{0} \frac{1}{z} c_{\text {cat }(\operatorname{surf})} c_{\text {solv }(\operatorname{surf})}}
$$

With degradation of this type it is very important to know the state of the catalyst solutions on the surface of the polymer, i.e, what the values of $c_{\text {cat(surf) }}$ and $c_{\text {solv(surf) }}$ are by themselves.

When an electrolyte solution is in contact with a solid polymer, the electrolyte may be in solution or in the polymer or on the phase interface (solution-polymer). The state of the electrolyte in the solution and in the polymer is assessed in literature. The concentration and activity gradients which may occur at the solution-polymer interface are brought about by the occurrence of degradation, including the diffusion of the electrolyte and of the reaction products and the chemical reaction, which disturbs the equilibrium of statistical distribution of the electrolyte (molecules and ions) at the surface of the polymer specimen (a concentration effect), and also by the fact that the electrolyte and reaction products can be adsorbed on the surface of the polymer specimen (an adsorption effect).

The concentration effect was investigated in detail mostly by McGregor and Peters [15], who established a link between the thickness of the surface layer $\delta$ and the diffusion parameters of the substances in solution and in the polymer. They showed that at high rates of transport of the solution $\delta \rightarrow 0$.

Thus, by using the transport of the solution, it is possible to reduce the concentration effect to a minimum.

The adsorption effect can be assessed from the Gibbs equation [16]:

$$
G=-\frac{a}{R T}\left(\frac{\partial \sigma}{\partial a}\right)_{T}
$$

where $G$ is the relative adsorption and $\sigma$ the surface tension at the polymer-solution interface.

The value of $\sigma$ is found from the Young equation:

$$
\cos \theta=\frac{\sigma_{p g}-\sigma}{\sigma_{s g}}
$$

where $\sigma_{p g}$ and $\sigma_{s g}$ are, respectively, the surface tensions at the polymer-gas and solution-gas interfaces, and $\theta$ the wetting angle.

Aqueous electrolyte solutions are surface-inactive and the value of $G$ is negative. Calculations for hydrophobic polymers show that $G$ is low and that, in the majority of cases, it can be assumed that the thermodynamic parameters of the catalyst and solvent will be closely similar in solution and at the polymer-solution interface.
The physicochemical basis of the biodegradation of synthetic polymers and of the resorption of polymer implants is given by Livshits [17]. The equations which describe the external diffusion-kinetic conditions are considered.

Aqueous electrolyte solutions are surface-inactive and the value of $G$ is negative. Calculations for hydrophobic polymers show that $G$ is low and that, in the majority of cases, it can be assumed that the thermodynamic parameters of the catalyst and the solvent will be closely similar in solution and at the polymer-solution interface.

The physicochemical basis of the biodegradation of synthetic polymers and of the resorption of polymer implants is given by Livshits [17]. The equations which describe the external diffusion-kinetic conditions are considered.

\section{Special Features of Degradation of Polymer Articles of Inhomogeneous Structure}

In polymer articles there is practically always anisotropy of structural features and properties throughout the volume (the so-called structural differentials [18]).

In single-phase systems there are the following examples of such differentials:

- glass-like and high-elastic portions (copolymers of methyl methacrylate and methylacrylate);

-heavily and lightly crosslinked regions (vulcanizates and elastomers);

- orientated and nonorientated regions (crystallizing polymers);

- regions with differing MMDs (fractionation under shear flow);

- regions within single-phase amorphous polymers with differing degrees of ordering (association).

In hetero-phase systems the following major structural differentials occur:

- amorphous and crystalline regions (crystallizing polymers);

- regions with differing degrees of microinhomogeneity (stereoregular polymers);

- regions with differing three-dimensional structure (cis-trans isomerism) or chemical structure.

In addition, in polymeric materials there are varied structural differentials created by additions of organic and inorganic substances, such as stabilizers, plasticizers, blowing agents, fillers (glass fibers in glass reinforced plastics and carbon black in rubber), pigments, etc.

These structure differentials bring about anisotropy of chemical and diffusion properties in polymers articles. At the present time there is a lack of precise quantitative data to show the influence of structural factors on the diffusion of aggressive media on the one hand and on the 


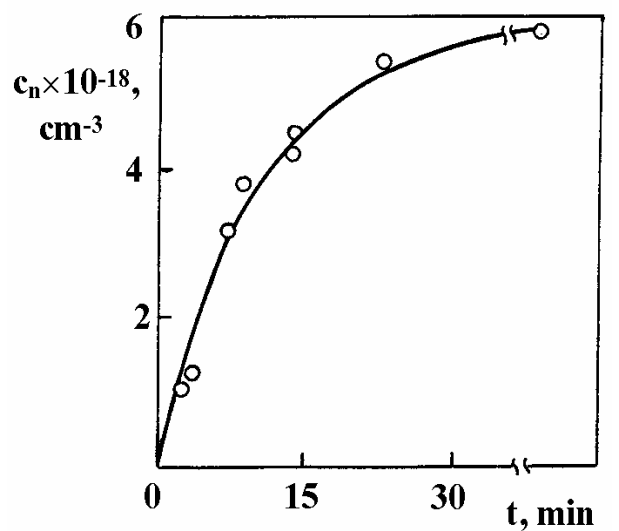

Fig. 1. Kinetics of accumulation of carboxyl groups formed in the hydrolytic degradation of PK-4 polycaproamide film at $333 \mathrm{~K}$ in water [21]

reactivity of the functional groups in the polymers on the other hand. This is discussed more fully in $[19,20]$.

Generally, in a polymer there are regions with differing reactivity (differing $k$ values) and different capacity for sorbing and transporting aggressive media (differing $c^{0}$ and $D$ ).
Thus, the overall rate of the degradation process is

$$
w_{\Sigma}=\sum_{t}\left[k\left(c_{n}^{0}-c_{n}\right) c_{c a t} c_{\text {solv }}\right] v_{i}
$$

where $v_{i}$ is the relative volume of each "region".

Equation (41) shows that degradation can occur at differing rates in different parts of a polymer article, i.e. there is particular "stepwise" chemical degradation in solid polymers.

This effect was first described in [21] for radical reactions in the solid phase. Under isothermal conditions there is an apparent overstepping of the reaction rate at a certain degree of dissociation of the total number of chemically unstable groups, while with the increase of the temperature the reaction begins afresh and finishes at a higher degree of dissociation.

Fig. 1 shows the kinetic curve for the accumulation of carboxyl groups which are formed during the hydrolytic degradation of a film of polycaproamide at $333 \mathrm{~K}$ in water. Under these conditions less than 12 of all the amide groups decompose [22]. At a higher temperature (> $423 \mathrm{~K}$ ) to a perceptible degree decomposition of most of the remaining amide groups within the same time takes place.

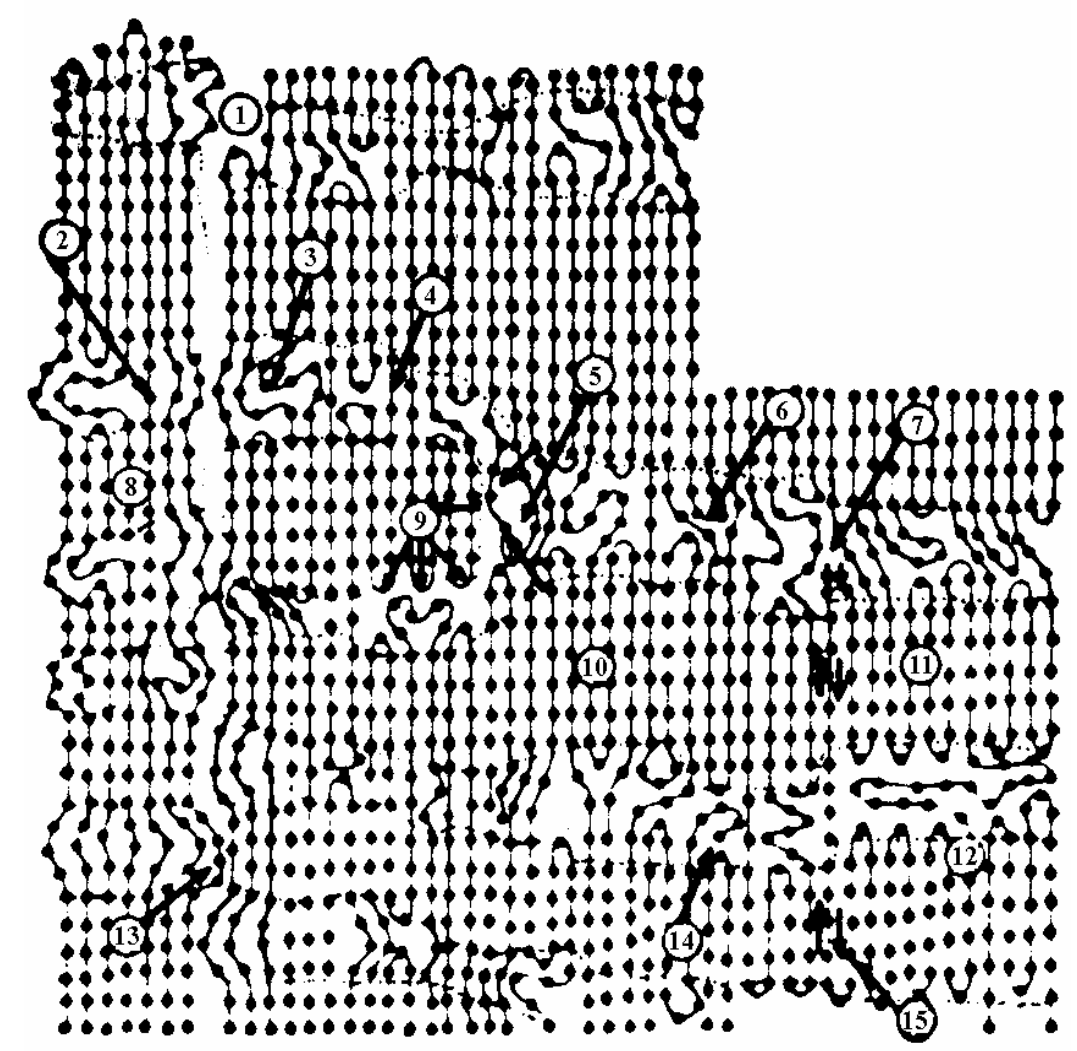

Fig. 2. Faulty structure of macromolecular crystals [22]: voids (1), the Statton model (2), amorphous regions (3), straight chains (4), crystallites of block material (5), a long fold (as proposed by Flory) (6), a migrating fold (7), individual fibrils (cold drawing) (8), a short fold (as proposed by Keller) (9), paracrystalline laminar lattice (10), cluster fibrils (hot drawing)

(11), monocrystals (12), four-point pattern (13), chain end (14) and region of slipping (15) 
Let us consider the principal causes of stepwise chemical degradation.

\subsection{Accessibility of Chemically Unstable Groups in Polymers}

Generally, in regions of the polymer with differing structural differentials there may be differing concentrations of the components of the aggressive medium $\left(c_{\text {cat }}\right.$ and $\left.c_{\text {solv }}\right)$ around the chemically unstable groups, which leads to differing rates of chemical degradation. Moreover, it may be that the rate constants of decomposition of the chemically unstable groups in these regions will differ. An example of how $k$ differs considerably within a single region is considered below.

Let us consider crystallizing polymers. One possible model of a partially crystalline oriented polymer is shown in Fig. 2, which presents different types of ordering and disordering of segments of the macromolecule within the volume [23].

In the amorphous regions the microvoids are larger and, consequently, a considerably higher average concentration of the components of the aggressive medium than in the ordered regions may be set up.

All the functional groups in the polymer, including the chemically unstable groups, may be divided conventionally into "accessible" and "inaccessible" in relation to the first functional groups, the concentration of the components of the aggressive medium around these being much higher than around the other functional groups.

Fig. 2 shows that to "accessible" groups we can refer not only functional groups in the amorphous regions but also groups on the surface of the lamellae.

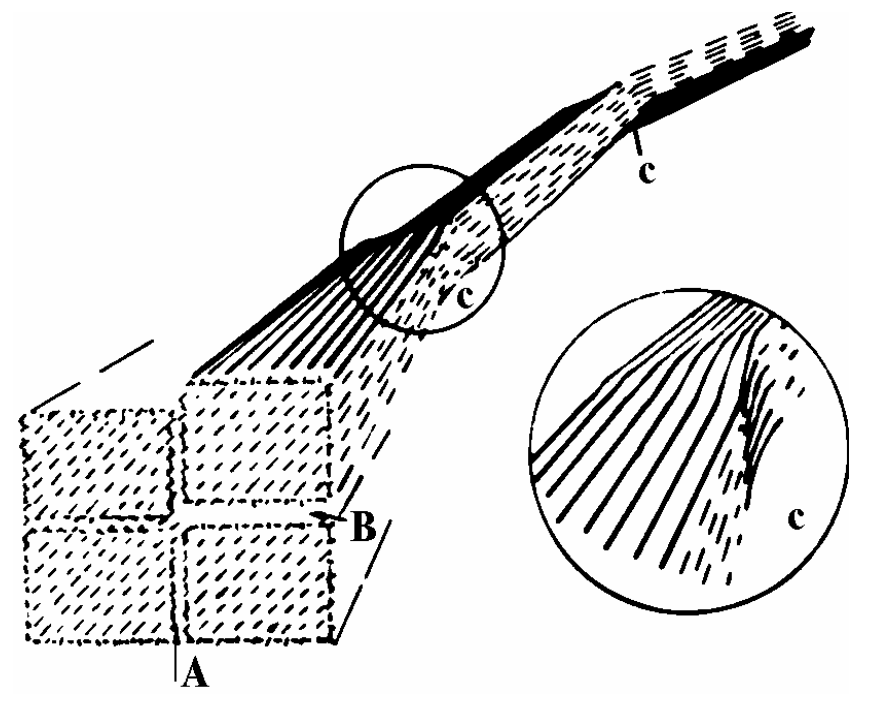

Fig. 3. Schematic representation of the surfaces of crystalline and nonordered segments of cellulose fibrils [26]
Quantitatively the "accessibility" of the functional groups in the polymer is expressed as follows:

$$
d=\frac{n}{n_{0}}
$$

where $n_{0}$ and $n$ are, respectively, the total amount of the functional groups and the amount of accessible groups.

Accessibility is always defined in relation to a particular reagent. For instance, within the same region the functional groups of a polymer may react with water molecules and not react with certain acid molecules. It is thus natural to expect that $d$ depends not only on the nature of the reagent but also on the external conditions.

Let us consider some examples of determination of the accessibility of the functional groups of the polymer.

First-order rate constants of formation of various substituted $\mathrm{O}$-glucopyranose residues in acid-catalyzed hydrolysis of cellulose at $298 \mathrm{~K}$ in $2.5 \mathrm{~N}$ HCI

\begin{tabular}{|l|l|l|l|l|l|}
\hline \multirow{2}{*}{$\begin{array}{c}\text { Glucopy- } \\
\text { ranose }\end{array}$} & $\begin{array}{c}\text { Non- } \\
\text { ordered } \\
\text { regments }\end{array}$ & $\begin{array}{c}\text { Crystal- } \\
\text { line } \\
\text { segments }\end{array}$ & $\begin{array}{c}\text { Glucopy- } \\
\text { ranose } \\
\text { residues }\end{array}$ & $\begin{array}{c}\text { Non- } \\
\text { ordered } \\
\text { segments }\end{array}$ & $\begin{array}{c}\text { Crystal- } \\
\text { line } \\
\text { segments }\end{array}$ \\
\cline { 2 - 5 } & $k \cdot 10^{3}, \mathrm{~s}^{-1}$ & $k \cdot 10^{6}, \mathrm{~s}^{-1}$ & & $k \cdot 10^{3}, \mathrm{~s}^{-1}$ & $k \cdot 10^{6}, \mathrm{~s}^{-1}$ \\
\hline $\begin{array}{l}\text { Unsubs- } \\
\text { tituted } \\
\text { 2-O-subs- } \\
\text { tituted }\end{array}$ & 1.1 & 6.5 & $\begin{array}{l}3-\mathrm{O}-\text {-subs- } \\
\text { tituted } \\
6-8\end{array}$ & 1.9 & 38.6 \\
\hline
\end{tabular}

Our investigation [24] connects the influence of the structure of cellulose on the accessibility of glucose groups with acid-catalyzed degradation.

In [25] there is a detailed study of the structure of the accessible regions in cellulose. A quite small amount of diethylaminoethyl substituent was added to cellulose, which caused practically no change in the structure of the polymer (as shown by X-ray diffraction analysis) and which was easy to determine quantitatively in soluble and insoluble products of acid-catalyzed hydrolysis by means of GLC. This method made it possible to determine the presence of O-diethylaminoethyl groups in 2, 3, and 6 positions of the D-glucopyranose residues.

The hydrolysis reaction takes place on the surface of the fibrils; the authors distinguished two types of accessible surfaces which they call crystalline and nonordered segments.

Table 1 shows the effective first-order rate constants of the formation of various substituted Dglucopyranose residues during the course of acid-catalyzed hydrolysis of cellulose at $298 \mathrm{~K}$ in $2.5 \mathrm{~N} \mathrm{HCI}$.

This difference in the reactivity of the glycoside group is brought about, according to Miller [26], by the higher accessibility of the groups for protonation in the nonordered segments and greater ease of transition from 

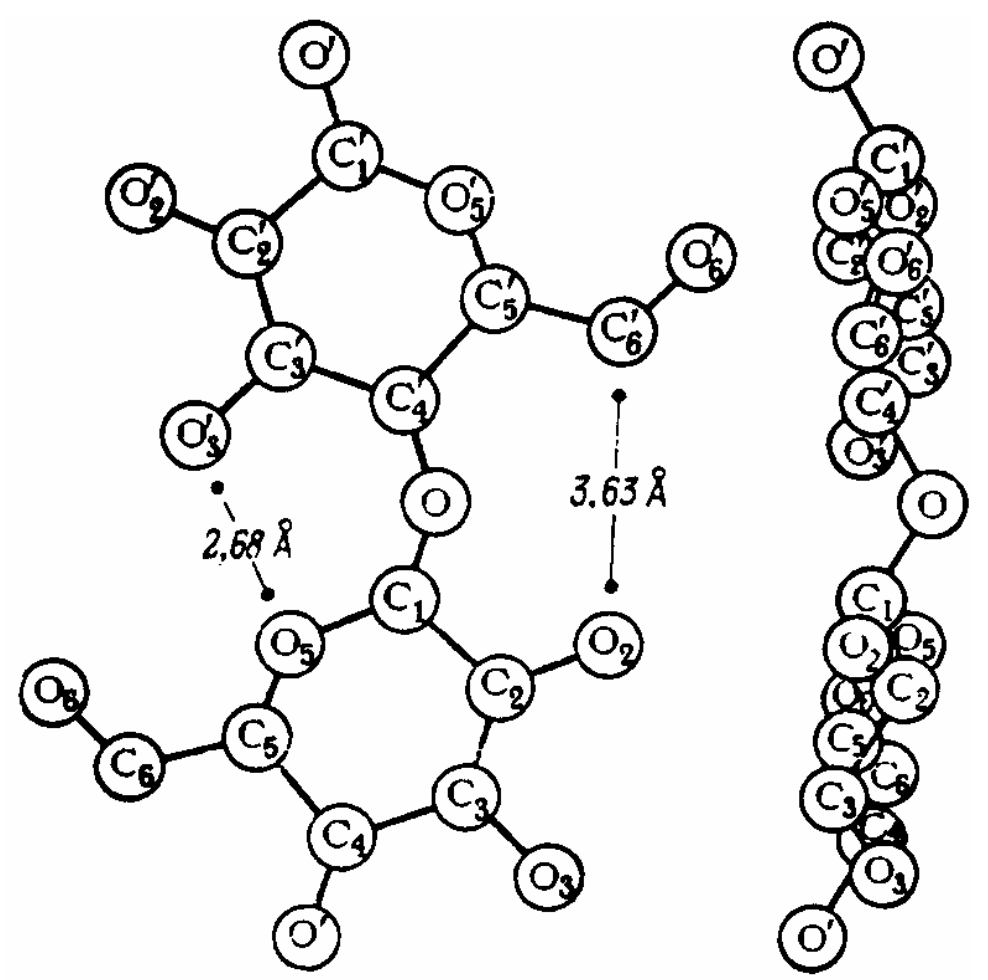

Fig. 4. Conformation of a cellobiose segment of a crystalline cellulose chain [26]

the chair conformation to the half-chair conformation (the limiting stage of the hydrolysis reaction).

The distribution of accessible surface between nonordered and crystalline segments has been determined from the data on distribution of 2-O-substituted D-glucopyranose residues in these regions. This ratio is $0.26: 0.74$ in the initial period of reaction; it changes in the course of hydrolysis to $0.36: 0.64$ because of recrystallization of the cellulose.

Surfaces of crystalline and nonordered segments of fibrils are shown diagrammatically in Fig. 3. In the crystalline regions (surfaces $\mathrm{A}$ and $\mathrm{B}$ ) the segments are oriented and the hydroxyl groups are in regularly repeating positions, with the hydroxyl group in position 3 linked by a strong hydrogen bond to the oxygen in the ring of the adjacent D-glucopyranose residue (Fig. 4). The main part of the defects of the crystalline structure caused by twisting, flexure of the fibrils, and internal stresses are concentrated in the non-ordered regions (surface $\mathrm{C}$ ). The hydrogen bonds $\mathrm{O}_{3}{ }^{\prime}-\mathrm{H}-\mathrm{O}_{5}$ in these regions are the least strong, and this increases the rate constant of formation of 3-O-substituted glucopyranose residues.

For polymers with labile hydrogen atoms one convenient method of determining the accessibility of the functional groups is the reaction of H-D exchange since in the course of this reaction the concentration of functional groups remains unchanged.
In [27], using the change in absorption intensity at $2480 \mathrm{~cm}^{-1}$ (VND) (IR spectroscopy), the accessibility of the amide bonds in a film of polycaproamide in relation to $\mathrm{D}_{2} \mathrm{O}$ was determined. Fig. 5 shows how $d$ changes with temperature. With increase of the temperature from 298 to $353 \mathrm{~K}$ the accessibility increases and becomes greater than the proportion of the amorphous phase (the degree of crystallinity was determined by X-ray diffraction analysis). The result may be explained by deuteration of

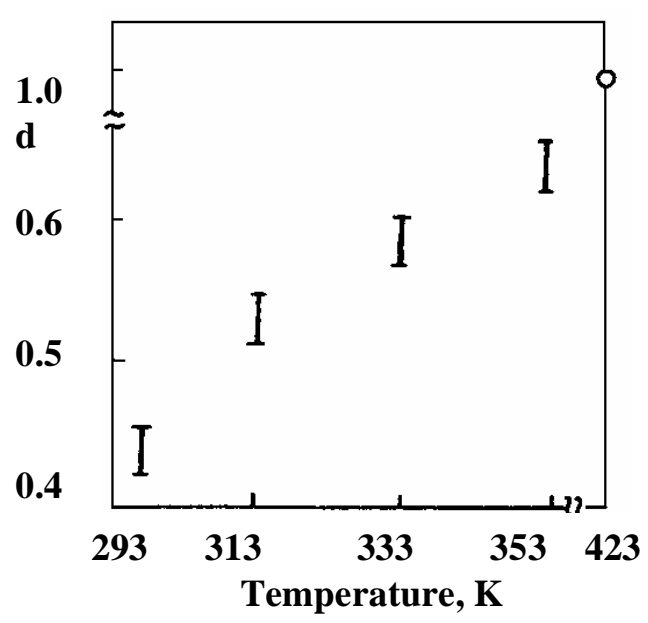

Fig. 5. Accessibility of amide groups $d$ in PK-4 polycaproamide as a function of temperature 
the amide groups in the faulty structures on the surface of the crystalline formations.

At $423 \mathrm{~K}$ all the amide groups, including those in the ordered regions, are accessible to $\mathrm{D}_{2} \mathrm{O}$ [28].

\subsection{Reactivity of Chemically Unstable Groups in Polymer Articles}

Generally, chemically unstable groups in different structural regions must have differing reactivity.

In amorphous polymers in which the accessibility of the functional groups is, as a rule, practically identical, the differences in the values of $k$ are not essential for different regions of the polymer, and we may therefore use the parameter of the mean rate of the degradation process, which is equal to the ratio of the overall rate of the process to the total volume of the polymer article:

$$
\bar{w}=\frac{\sum_{i}\left\lfloor k\left(C_{n}^{0}-c_{n}\right) c_{c a t} c_{s o l v}\right\rfloor_{i} V_{i}}{\sum_{i} V_{i}}
$$

In crystalline polymers there may be, even among the accessible groups, unstable groups with differing reactivity. For instance, in cellulose there are two types of glucoside bonds, differing in reactivity toward acidcatalyzed degradation: highly reactive glucoside bonds in the tips of the folds and those in the linear portions of the folds [29].

\section{Physical Model of the Process of Degradation of Polymer Articles}

The investigation of the kinetics of degradation of polymer articles in aggressive media involves great difficulties since the reaction takes place in the condensed phase and the reagents (the components of the aggressive medium and the chemically unstable groups of the macromolecules) are in close contact with the surrounding fragments of the macromolecules and the low-molecular particles.

Although at present there is a lack of precise molecular theories of electrolyte solutions in solid polymers, in some cases there are a number of semiempirical models which make it possible, at the molecular level, to consider approximately the kinetics of the degradation reactions of chemically unstable groups in polymers.

Let us consider the simplest degradation reaction; the interaction of chemically unstable groups of a polymer $R$ with a catalyst $c a t$. With the collision of $R$ and cat in the condensed phase these particles will collide with one another for a more or less prolonged time. The average lifetime of such a pair can be determined approximately from the theory of random processes [30, p. 423]:

$$
\bar{\tau} \approx \frac{2 r^{2}}{6 D_{\text {cat }}} \exp \left(-\frac{E_{S}}{k T}\right)
$$

where $r$ is the distance between $R$ and cat in the couple, and $E_{S}$ is the energy necessary to remove cat from $R$ by a distance $1.7 r$ in medium $S$.

For $E_{S}=0$, we can estimate the limiting value of $\bar{\tau}$ in solution and within the matrix of a solid polymer for typical values of $D_{c a t}$ in these media, assuming $r=(4 \pm 1) \stackrel{\mathrm{C}}{\mathrm{A}}$ :

\begin{tabular}{|l|c|c|}
\hline & $D_{c a t}, \mathrm{~cm}^{2} / \mathrm{s}$ & $\bar{\tau}, \mathrm{s}^{-1}$ \\
\hline Solution & $10^{-5}$ & $10^{-11}$ \\
\hline Polymer & $10^{-8}$ & $10^{-8}$ \\
\hline
\end{tabular}

From the data it may be seen that within the matrix of the solid polymer the average lifetime of the couple $R$ and cat is quite long, and in the couple equilibrium for all degrees of freedom is established, i.e., these couples can be regarded as kinetically independent particles. The specific kinetics of bimolecular reactions in a matrix of a solid polymer are considered in $[21,31]$. In the event of only two stages present microdiffusion and kinetic (the catalyst and chemically unstable groups in the polymer article being distributed uniformly), chemical interaction between cat and $R$ begins only when they form a couple in microvoids of volume $V^{*}$.

As a result of any external activation cat may move relative to $R$ at rate $k_{t}$, but in couples it may react with constant $k_{p}$.

Under steady-state conditions (the rates of formation and of destruction of couples being equal) the kinetic equation for the rate takes the following form

$$
\frac{-d c_{d}}{d t}=k c_{m} c_{c a t}
$$

where $k=\frac{k_{t} k_{p}}{k_{t}+k_{p}} V^{*}$

For $k_{t} \gg k_{p}$ the kinetic stage is the limiting one and

$$
k=k_{p} V^{*}
$$

For $k_{t}<<k_{p}$, the microdiffusion stage (the frequency of approach) is the limiting one

$$
k=k_{t} V^{*}
$$


The movement of cat and $R$ in the polymer matrix is effected as a cooperative process. Accordingly the kinetics of the degradation reactions proves to be linked with the kinetics of the molecular movements, with the physical properties, and with the structure of the polymer.

The character of this relationship is such that the processes of structural relaxation of the macromolecules within the matrix of a solid polymer are hindered, and within the time of an elementary process of the chemical reaction it is not always possible to bring about the most favorable state of the transition complex, i.e., the elementary reactions take place over a higher potential barrier than the same reactions in liquids.

Such ideas were first put forth by Kooyman and Strang [32]. Now there are a number of investigations confirming this postulate.

In [33] a correlation is found between the rate constant of the bimolecular reaction $k$ (the interaction of 2,4,6-tert-butylphenol with hydrogen peroxide groups in polyolefins) and the frequency of rotation $v$ of the stable nitrosyl radical; this frequency characterizes the mobility of the macromolecules in polymers with differing solvent content. For the reaction of a phenoxy radical with a hydrogen peroxide group it is necessary for the reagents to assume the necessary configuration and to have sufficient energy. The movement of the reagents takes place within the microvoids, and the greater the "stiffness" of the walls of the microvoids the higher the energy which has to be expended for a chemical reaction to take place.

The rate constant of the reaction within the matrix of a solid polymer may be presented as follows:

$$
k=p_{\text {prob }} p \exp \left(-\frac{E_{\text {prob }}+E}{R T}\right)
$$

where $p$ and $E$ are, respectively, the steric factor and the activation energy in the gaseous phase, and $p_{\text {prob }} \exp \left(-\frac{E_{p r o b}}{R T}\right)$ the probability of rotation of the reagents in the condensed phase for creation of the activated complex configuration.

With increase of the solvent concentration in the polymer there is an increase in the size of the microvoids due to the solvent molecules and reduction in the "stiffness" of their walls. These two effects lead to a considerable rise in the rate constant and reduction in the activation energy of the chemical reaction. With a solvent content in the polymer around 40 mas $\%, \mathrm{~V}$ and $k$ cease to depend on the solvent content in the matrix of the polymer and, hence, become equal to the corresponding figures in pure solvent (the number of solvent molecules in the microvoids is sufficient to screen the reagents from the influence of the walls of the microvoids).

It should be noted that in the reactions of chemically unstable groups with aggressive media ion pairs and ions can participate as reagents; where such reactions occur the kinetic parameters may be significantly influenced by the microdielectric permittivity and the specific interactions in the microvoids.

In polymers, at temperatures below $T_{g}$ there are micropores of various sizes which, as already indicated

Features of the occurrence of chemical degradation

Table 2

\begin{tabular}{|c|c|c|c|}
\hline Features & Internal diffusion-kinetic region & Internal kinetic region & $\begin{array}{l}\text { External diffusion-kinetic } \\
\text { region }\end{array}$ \\
\hline $\begin{array}{l}\text { Molecular mass of the } \\
\text { polymer (film, thread, } \\
\text { section from surface of } \\
\text { polymer) }\end{array}$ & Changes & Changes & $\begin{array}{c}\text { Remains practically } \\
\text { unchanged }\end{array}$ \\
\hline \multirow{4}{*}{$\begin{array}{l}\text { Dependence of the rate of } \\
\text { degradation on: } \\
\text { the surface of the specimen } \\
\text { the volume of the specimen } \\
\text { the structure differentials }\end{array}$} & & & \\
\hline & Is dependent & Is independent & Is dependent \\
\hline & Is practically independent & Is dependent & Is independent \\
\hline & $\begin{array}{l}\text { Is dependent, change in } \\
\text { accessibility, } c_{c a t} \text { and } c_{\text {solv }}\end{array}$ & $\begin{array}{l}\text { Is dependent, change in } \\
\text { accessibility, } c_{c a t}^{0} \text { and } c_{\text {solv }}\end{array}$ & Is practically independent \\
\hline $\begin{array}{l}\text { concentration (or activity) } \\
\text { of the catalyst and solvent }\end{array}$ & Depends on $c_{c a t}$ and $c_{\text {solv }}$ & Depends on $c_{c a t}^{0}$ and $c_{\text {solv }}$ & $\begin{array}{l}\text { Depends on } h_{x} \text { and } \alpha_{s o l v} \\
\text { in the solution }\end{array}$ \\
\hline
\end{tabular}


in the literature, influence the sorption and permeability of the components of the aggressive medium, which, in turn, considerably raises the rate of degradation on the surface of the micropores.

All these factors can influence the kinetic parameters in differing ways, making the dependence of $k$ on the solvent content of the polymers often more complex than in the example given above.

Thus, degradation of polymer articles in aggressive media can take place in the following regions:

- an internal diffusion-kinetic region (degradation takes place in a reaction zone whose size increases with time and ultimately attains the dimensions of the polymer article);

- an internal kinetic region (degradation takes place at an identical rate throughout the volume of the polymer article);

- an external diffusion-kinetic region (degradation takes place in a thin surface layer of constant dimension, which at the limit can be a monolayer of the polymer).

Table 2 shows the characteristic features of the occurrence of chemical degradation of polymers in each of the three regions.

Where these processes take place in the internal diffusion-kinetic and internal kinetic regions, it is necessary, when determining the kinetic parameters, to take into account the differences in accessibility and in reactivity of the chemically unstable groups in the polymer articles [34, 35].

\section{References}

[1] Emanuel N. and Buchachenko A.: Chemical physics of degradation and stabilization of polymers. VSP International Science Publ., Utrecht 1982.

[2] Bochkov A. and Zaikov G.: Chemistry of the glycosidic bonds. Formation and cleavage. Pergamon Press, Oxford 1979. [3] Razumovskii S. and Zaikov G.: Ozone and its reactions with organic compounds. Elsevier, Amsterdam 1984.

[4] Emanuel N., Zaikov G. and Maizus Z.: Oxidation of organic compounds. Medium effects in radical reactions. Pergamon Press, Oxford 1984.

[5] Afanasiev V. and Zaikov G.: V oblasti kataliza. Mir, Moskva 1979.

[6] Moiseev Yu. and Zaikov G.: Chemical resistance of polymers in reactive media. Plenum Press, New York 1987.

[7] Zaikov G., Iordanskii A. and Markin V.: Diffusion of electrolytes in polymers. VNU Science Press, Utrecht 1988.

[8] Minsker K., Kolesov S. and Zaikov G.: Degradation and stabilization of polymers on the base of vinylchloride. Pergamon Press, Oxford 1988.

[9] Aseeva R. and Zaikov G.: Combustion of polymer materials. Karl Hanser Verlag, Munchen 1986.

[10] Popov A., Rapoport N. and Zaikov G.: Oxidation of stressed polymers. Gordon \& Breach, New York 1991.
[11] Entelis S. and Tiger R.: Kinetika reakcij v gydkoi phase. Khimiya, Moskva 1973.

[12] Danckwerts P.: Trans. Faraday Soc., 1950, 46, 300.

[13] Carslow H. and Jaeger J.: Conduction of heat in solids. Oxford University Press, London 1947.

[14] Crank J.: The mathematics of diffusion. Oxford University Press, London 1956.

[15] McGregor R. and Peters R.: J. Soc. Dyers Colour, 1965, 81, 393.

[16] Fridriksberg D.: Kurs koloidnoi khimii. Khimiya, Leningrad 1974.

[17] Livshits V.: Vysokomol. Soedin. A, 1972, 14, 394.

[18] Shen M. and Sever M.: J. Mater. Sci., 1972, 7, 741.

[19] Lososki S. and Cobbs W.: J. Polym. Sci., 1959, 36, 21.

[20] Vatrow G., Ravens D. and Ward J.: Polymer, 1962, 3, 17.

[21] Lebedev Ya.: Kinet. Katal., 1967, 8, 245.

[22] Bershtein V. and Egorova L.: Vysokomol. Soedin. A, 1977, 19, 1260.

[23] Hosemann R.: J. Appl. Phys., 1963, 34, 25.

[24] Haworth S. et al.: Carbohydr. Res., 1969, 10, 1.

[25] Rowland S. and Roberts E.: J. Polym. Sci. A-1, 1972, 10, 2447.

[26] Be Miller J.: Adv. Carbohydr. Chem., 1967, 22, 25.

[27] Markin V.: PhD thesis, Moscow 1974.

[28] Schmidt P. and Schneider B.: Collect. Czech. Chem. Commun., 1966, 31, 1896.

[29] Chang M., Pound T. and Manley R.: J. Polym. Sci., 1973, 11, 399.

[30] Benson S.: Foundations of chemical kinetics, McGrawHill, New York 1960.

[31] Pudov V. and Buchachenko A.: Uspehi Khim., 1970, 39, 130.

[32] Kooyman E. and Strang A.: Recl. Trav. Chim., 1953, 72, 329.

[33] Griva A. and Denisov E.: Dokl. Akad. Nauk SSSR, 1974, 219, 640.

[34] Polishchuk Ya. and Zaikov G.: Multicomponent transport in polymer system for controllable release. Gordon and Breach, Newark 1996.

[35] Iordanski A., Rudakova T. and Zaikov G.: Interaction between polymers and chemical and biological media, VSP Sci. Publ., Zeist 1995.

\section{РОЗКЛАД ПОЛІМЕРІВ В АГРЕСИВНОМУ СЕРЕДОВИЩІ. КІНЕТИЧНИЙ ПІДХІД}

Анотація. Показано, щзо розклад полімерів в агресивному середовищі є складним фізико-хімічним процесом, який включає в себе адсорбиію, дифузію і дисоціацию хімічно нестабільних зв'язків. Встановлено, що на розклад впливає як будова полімерних матеріалів, так $i$ кінетика реакиій у твердому стані.

Ключові слова: розклад, стабілізація, агресивне середовище, вуглеланиюгові ігетероланиюгові полімери, хімічна кінетика. 